\title{
Rapid room-temperature synthesis of hierarchical porous zeolitic imidazolate frameworks with high space-time yield
}

\author{
Chongxiong Duan, Feier Li, Jing Xiao, Zewei Liu, Chao Li ${ }^{*}$ and Hongxia Xi ${ }^{*}$
}

\begin{abstract}
Hierarchical porous zeolitic imidazolate frameworks (ZIFs) have potential for adsorption, catalysis and chemical sensing applications. Ultrafast synthesis of ZIFs at room temperature and pressure is particularly desirable for large-scale industrial production. Here, we developed a green and versatile method using organic amines as supramolecular templates (organic amine-template) to rapidly synthesize hierarchical porous ZIFs (ZIF-8, ZIF-61 and ZIF-90) at room temperature and pressure. The synthesis time was reduced dramatically to within $1 \mathrm{~min}$, and the resulting ZIFs had multimodal hierarchical porous structures with mesopores/ macropores interconnected with micropores. Notably, the space-time yield (STY) of hierarchical porous ZIF-8 was up to $1.29 \times 10^{4} \mathrm{~kg} \mathrm{~m}^{-3} \mathrm{~d}^{-1}$, which is more than three times higher than that reported using other methods. Furthermore, the morphologies and porosities of the produced ZIFs could be readily tuned by controlling the synthesis time or type of organic amine. The organic amine played two roles in the synthesis: (1) a protonation agent to deprotonate organic ligands, facilitating the formation of ZIF crystals, and (2) an structure directing agent to direct mesopore/macropore formation. The resulting hierarchical porous ZIF-8 exhibited enhanced uptake capacities and diffusion rates for guest molecules relative to its microporous counterpart. This work provides a new direction for the green and efficient synthesis of various hierarchical porous ZIFs with high STYs for a wide range of applications.
\end{abstract}

Keywords: zeolitic imidazolate frameworks, green and rapid synthesis, hierarchical porous structure, tunable morphology and porosity, high space-time yield

\section{INTRODUCTION}

Zeolitic imidazolate frameworks (ZIFs), consisting of bivalent transition metals ( $\mathrm{Zn}$ or $\mathrm{Co}$ ) and imidazolate-based ligands, are a distinctive subfamily of porous metal organic frameworks (MOFs) with zeolite-type tetrahedral topologies [1]. Combining the advantages of MOFs and zeolites, including high surface areas, uniform pore structures, and excellent thermal and chemical stabilities [2], provides versatile materials with potential for a wide range of industrial applications, including adsorption and separation [3], chemical sensing [4], and catalysis [5]. However, three major challenges have hindered the widespread applications of ZIFs. First, most reported ZIFs possess only micropores with pore sizes of less than $2 \mathrm{~nm}$ [1]. Although this intrinsic microporosity is desirable for separation and transportation of small molecules [6], when large molecules or high concentrations of reactants are involved in the reaction, they cannot rapidly approach or leave the interior active sites owing to pore size-confinement [7]. Furthermore, slow diffusion and mass transfer could lead to the polymerization of byproducts or intermediate products [8], which would block the channels and quickly deactivate ZIF materials. Therefore, the exclusively microporous structures in ZIF materials greatly limit their applications. Second, ZIF synthesis is conventionally conducted solvothermally, by using a high-boiling solvent (e.g., N,N-dimethylformamide (DMF)) and taking a long time at high temperature and pressure (HTP) in an autoclave [9]. Such solvothermal processes usually consume huge amounts of energy and may lead to environment pollution or pose the risk of explosion [10]. The toughest challenge in ZIF synthesis is the low production rate [11]. For example, the space-time yield (STY) of ZIFs is usually below $100 \mathrm{~kg} \mathrm{~m}^{-3} \mathrm{~d}^{-1}$, which presents a considerable hurdle for the large-scale commercial production and industrial applications [12].

To date, extensive efforts have been made toward developing efficient methods to overcome the above chal-

School of Chemistry and Chemical Engineering, South China University of Technology, Guangzhou 510640, China

" Corresponding authors: (emails: chemlic@scut.edu.cn (Li C); cehxxi@scut.edu.cn (Xi H)) 


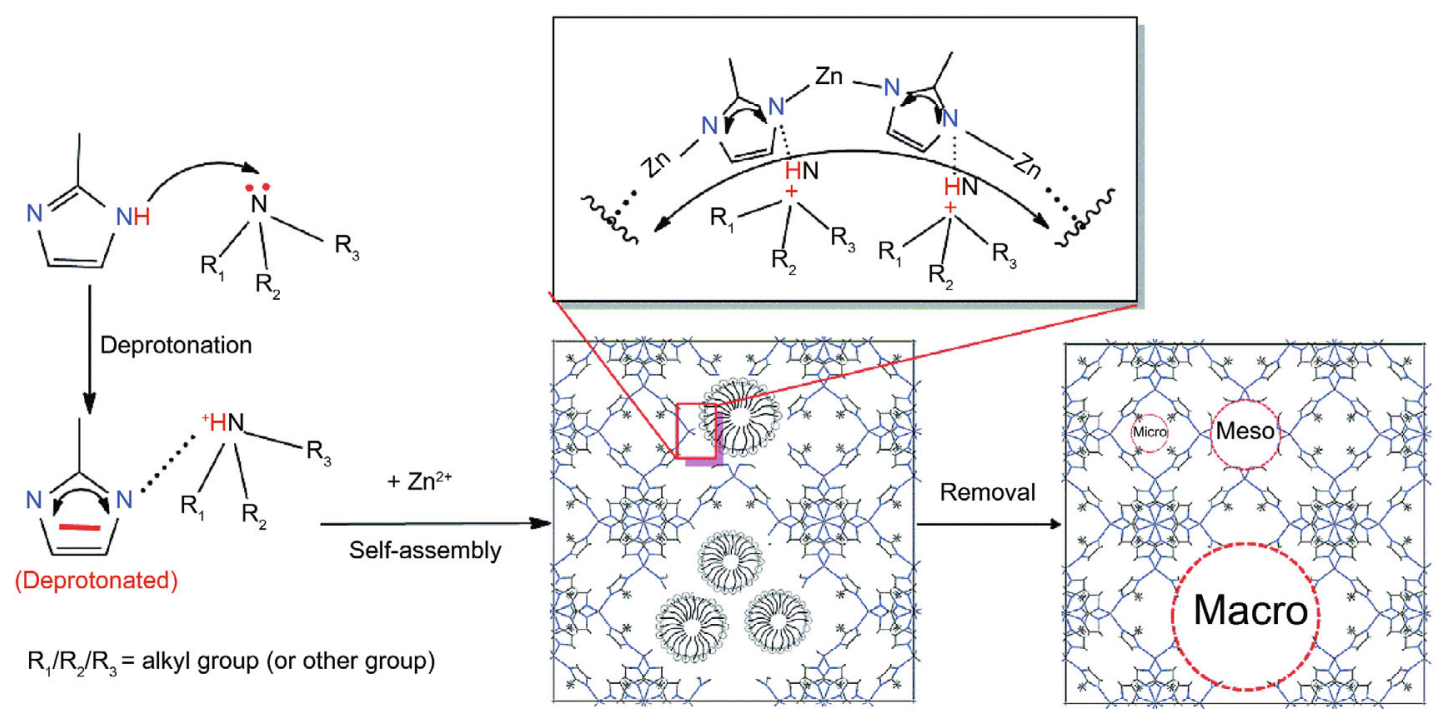

Scheme 1 Illustration of the supramolecular template concept for rapid synthesis of hierarchical porous ZIF-8 at room temperature and pressure.

lenges [13]. For example, to improve diffusion rates and facilitate mass transfer of guest molecules in ZIFs, some strategies were developed to increase the size of ZIF pores, including template [14], amino acid-assisted methods [15], and composite materials [16]. Although these approaches can successfully obtain desired hierarchical porous structures, the rational design of porous ZIFs with controllable/tailorable structures and porosities for specific applications remains challenging $[6,17]$. Moreover, these methods still consume too much energy $[11,18]$. In addition, a few advanced approaches, such as microwave-techniques [19], ultrasound-assisted methods [20], and mechano-chemical methods [21] have been employed to reduce energy consumption and enhance production rates. However, these approaches often require complex apparatus or set-ups [22], and more importantly, are still restricted to conventional ZIFs with micropores. Overall, a simple and efficient method for the room temperature and pressure (RTP) synthesis of hierarchical porous ZIFs with tunable porosities and high STY remains a formidable challenge.

Herein, we demonstrate a simple and green approach to rapidly synthesize hierarchical porous ZIF-8 materials within 1 min at RTP by using organic amines as a supramolecular template (organic amine-template). The obtained ZIF-8 products contained hierarchical porous structures with micro-, meso-, and macropores. Moreover, the morphology and porous structure could be easily tailored by varying the synthesis time or type of organic amines. Furthermore, our synthesis strategy can also be applied to the synthesis of a wide variety of other hierarchical porous ZIFs (e.g., ZIF-61 and ZIF-90). The resulted hierarchical porous ZIFs exhibited much higher uptake capacities and selectivity for gas molecules than pristine microporous materials. In addition, the STY for the ZIF-8 was up to $1.29 \times 10^{4} \mathrm{~kg} \mathrm{~m}^{-3} \mathrm{~d}^{-1}$, which is at least three times higher than the previously reported.

\section{RESULTS AND DISCUSSION}

Scheme 1 illustrates our supramolecular templating approach for rapidly synthesizing hierarchically porous ZIF8. Zinc nitrate $\left(\mathrm{Zn}\left(\mathrm{NO}_{3}\right)_{2}\right)$ methanol solution was added to a 2-methylimidazole (2-Im) methanol solution at RTP to form a transparent mixture solution. Milky precipitate generated quickly when an organic amine (Table S1) was added into the mixture solution under stirring (Video S1) - a definitive visual indication of ZIF-8 formation. The milky precipitate was immediately filtered after stirring for a given reaction time $t$, then washed with methanol and dried. The resulting products were labeled as ZIF8_A $t$, in which A means diethanolamine was used as the supramolecular templating, and $t(t=1,5,10$ or $30 \mathrm{~min})$ represents the reaction time in a few minutes (Supplementary Information).

\section{X-ray diffraction analysis of hierarchical porous ZIF-8}

Fig. 1 compares simulated and experimental X-ray diffraction (XRD) patterns for ZIF-8 powders obtained using rapid RTP synthesis and using conventional solvothermal synthesis for $24 \mathrm{~h}$ at $413 \mathrm{~K}$ [18]. As shown in 


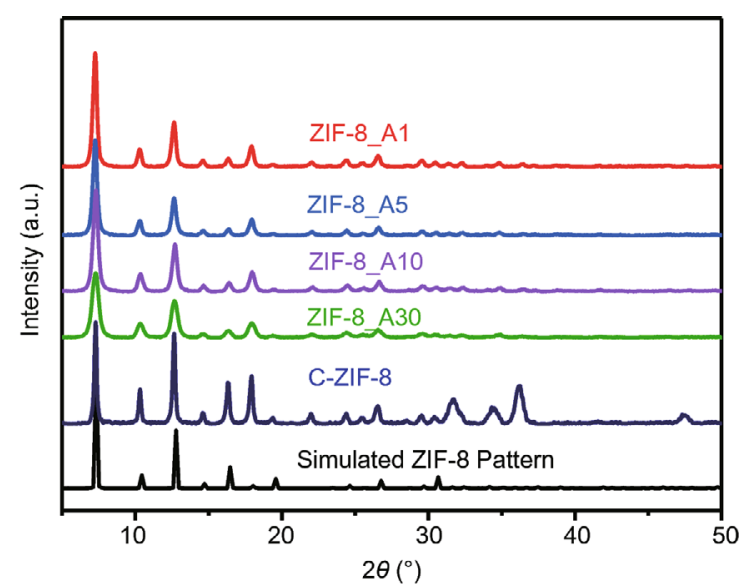

Figure 1 Powder XRD patterns of ZIF-8_At $(t=1,5,10$, or $30 \mathrm{~min})$ and conventional ZIF-8 (C-ZIF-8) samples, and simulated XRD pattern of ZIF-8.

Fig. 1, the positions of all the diffraction peaks for ZIF$8 \_$A $t(t=1,5,10$, or $30 \mathrm{~min})$ agree well with those for conventional ZIF-8 (C-ZIF-8) and the simulated results, confirming that ZIF- 8 crystals were successfully synthesized under RTP. Moreover, the sharp XRD peaks indicate that the ZIF-8_At samples have high crystallinity. Notably, the shortest synthesis time in our strategy was only $1 \mathrm{~min}$ at RTP, whereas conventional solvothermal methods require a synthesis time of $24 \mathrm{~h}$ at HTP [18], demonstrating that organic amines play a significant role in accelerating the formation of ZIF-8. Gross et al. [23], Nordin et al. [24], and Cravillon et al. [25] also reported that ZIFs could be synthesized within $10 \mathrm{~min}$ at RTP by using an organic amine (triethylamine) as a protonation agent, indicating that the organic amine (e.g., diethanolamine) used in this work acts as a protonation agent. However, the ZIFs synthesized with triethylamine as a protonation agent only possessed microporous structures instead of hierarchical porous structures. In addition, the reaction time in these previous methods $(10 \mathrm{~min})$ was longer than that in our strategy $(1 \mathrm{~min})$.

\section{Morphologies of hierarchical porous ZIF-8}

The crystal morphologies of ZIF-8_At $(t=1,5,10$, or 30$)$ and C-ZIF-8 were revealed by scanning electron microscopy (SEM) and transmission electron microscopy (TEM). The SEM images in Fig. 2 show that each ZIF8_At sample has a uniform crystal structure, unlike CZIF-8, which has an irregular shape (Fig. S1). The SEM image in Fig. 2a reveals that ZIF-8_A1 is composed of nearly spherical nanoparticles with a diameter of about $50 \mathrm{~nm}$. The particle size of ZIF-8_A1 is considerably

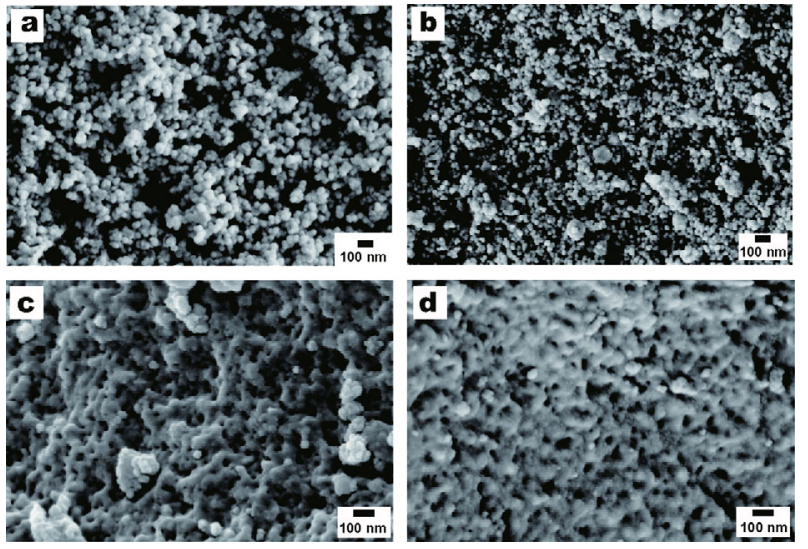

Figure 2 SEM images of hierarchical porous ZIF-8_A $t(t=1,5,10$, or 30 min): (a) ZIF-8_A1; (b) ZIF-8_A5; (c) ZIF-8_A10; (d) ZIF-8_A30.

smaller than that of a previous ZIF-8 sample synthesized with triethylamine within $10 \mathrm{~min}(\sim 100 \mathrm{~nm})$ or C-ZIF-8 (Fig. S1), [18,23] which can be attributed to fast nucleation $(<1 \mathrm{~min}$ ) between deprotonated ligands and metal ions, resulting in the formation of small nanocrystals $[23,25]$. Furthermore, the apparent voids surrounded by the walls consisting of nanoparticles (Fig. 2a) confirm the presence of a mesoporous structure. Hirai et al. [26], Kim et al. [27], and Cao et al. [28] also reported the formation of hierarchical pores between aggregated nanoparticles in ZIF materials. Moreover, when the synthesis time was extended, the small ZIF nanocrystals aggregated to form large grains. As shown in Fig. 2b, the nanoparticles obtained in ZIF-8_A5 are more compact. Furthermore, when the synthesis time exceeded $10 \mathrm{~min}$, similar morphologies were observed with well-ordered mesopores uniformly distributed on lumpy structures, as shown in Fig. 2c and d for ZIF-8_A10 and ZIF-8_A30, respectively. This trend can be attributed to continuous growth of the existing nanoparticles through the attachment of monomers in the presence of an organic amine template [29]. In addition, we note that the mesoporous structures of ZIF-8_A1 and ZIF-8_A5 primarily result from interparticle void space, whereas the crystals of ZIF8_A10 and ZIF-8_A30 are intrinsically mesoporous. These findings indicate that both the crystal morphologies and porosities of ZIF- 8 products can be influenced by the synthesis time.

The corresponding TEM images of the ZIF-8_A $t$ samples are presented in Fig. 3. As shown in Fig. 3a, numerous nanoparticles with diameters of about $50 \mathrm{~nm}$ are randomly stacked to form abundant mesoporous channels in ZIF-8_A1, with pore sizes of 30-100 nm according to the pore size distribution (PSD, Fig. 4b). 

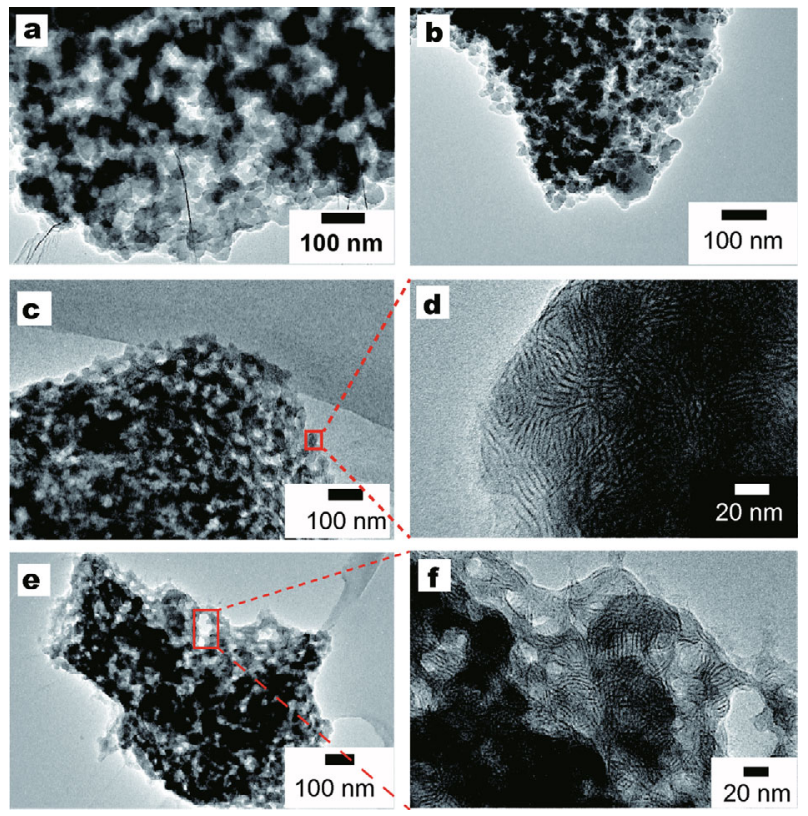

Figure 3 TEM images of hierarchical porous ZIF-8_At $(t=1,5,10$, or 30 min): (a) ZIF-8_A1; (b) ZIF-8_A5; (c) and (d) ZIF-8_A10; (e) and (f) ZIF-8_A30.

Hierarchical porous phases also exist between the aggregated nanoparticles in ZIF-8_A5, as shown in Fig. 3b. The TEM images of ZIF-8_A10 and ZIF-8_A30 (Fig. 3c, e) show similar morphologies with ordered lattice fringes. The lattice fringes can be clearly observed in high-resolution TEM (HR-TEM) images (Fig. 3d, f), confirming that these materials have high crystallinity [30]. These results indicate that the entire particle is crystalline with well-ordered mesopores penetrating through the microporous framework [31]. Thus, the introduced organic amines (surfactant) act as templates-termed structuredirecting agents (SDAs) that direct the formation of mesopores. Similar conclusions have been drawn by Bradshaw et al. [7], Wu et al. [15], and Junggeburth et al. [32], who used surfactants (neutral or ionic) as SDAs to synthesize hierarchical porous ZIFs.

\section{Porosities of hierarchical porous ZIF-8}

The hierarchical porosities of ZIF-8_A $t(t=1,5,10$, or 30 min) were further confirmed by examining their $\mathrm{N}_{2}$ adsorption-desorption isotherms (Fig. 4a) and nonlocal density functional theory (NL-DFT) PSDs (Fig. S2b and Fig. 4b). According to the IUPAC classification [33], CZIF-8 displays a type I isotherm (Fig. S2a) because it only possesses micropores, whereas ZIF-8_A $t(t=1,10$, or 30 min) exhibit a combination of type I and type IV isotherms with a typical hysteresis loop [34], and ZIF-8_A5 shows a type IV isotherm (Fig. 4a). These observations confirm the co-existence of micropores and mesopores in these ZIF-8_At materials. In addition, among the ZIF8_At samples, the adsorption capacities and hysteresis loop shapes depend on the synthesis time. Further information concerning the porosity of the ZIF-8_At samples can be acquired from the PSD (Fig. S2b). In addition to intrinsic micropores (pore diameter: $<2 \mathrm{~nm}$ ), all the ZIF-8_At samples show both mesopores (pore diameter: 2 50 nm) and macropores (pore diameter: $>50 \mathrm{~nm}$ ), as shown in Fig. S2b, which are not observed in C-ZIF-8 (inset of Fig. S2a). Although a couple of ZIF-8 materials have been reported with pore diameters in two ranges (micropores and mesopores) [15,32], to the best of our knowledge, no hierarchical porous ZIF-8 materials have been synthesized previously at RTP with pore sizes encompassing three ranges (micropores, mesopores, and macropores).

The development of reliable methods to synthesize hierarchical porous ZIFs with tunable porosities remains
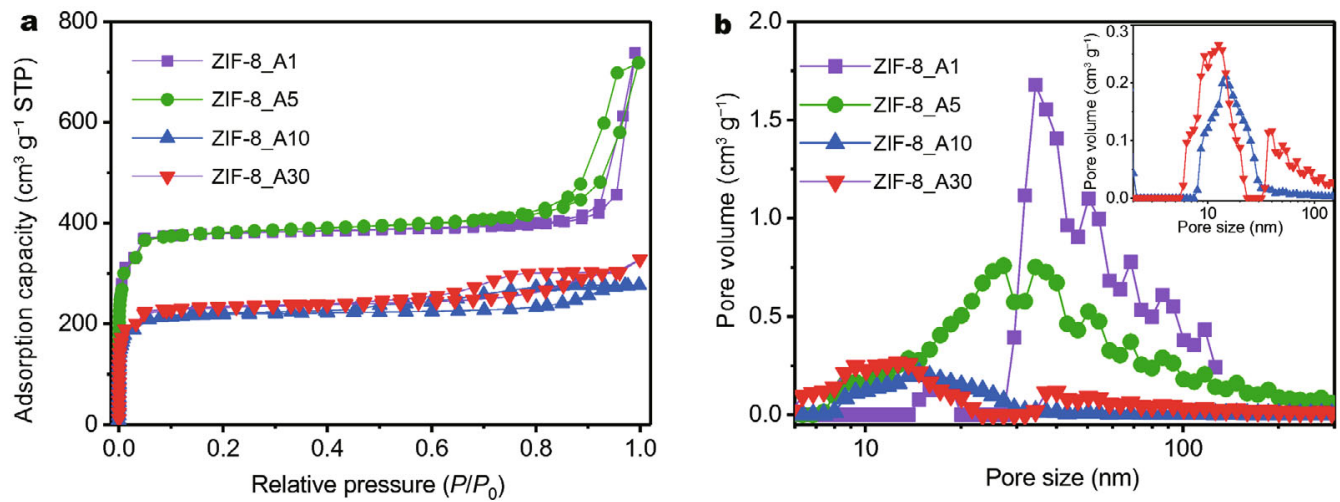

Figure 4 (a) $\mathrm{N}_{2}$ adsorption-desorption isotherms and (b) pore size distributions (PSDs) for hierarchical porous ZIF-8_A $t(t=1,5$, 10, or 30 min). 
Table 1 Porosities and STYs of the various ZIF-8 samples synthesized in this work

\begin{tabular}{|c|c|c|c|c|c|c|}
\hline Sample & Synthesis time & $S_{\text {BET }}\left(\mathrm{m}^{2} \mathrm{~g}^{-1}\right)^{\mathrm{a}}$ & $V_{\mathrm{t}}\left(\mathrm{cm}^{3} \mathrm{~g}^{-1}\right)^{\mathrm{b}}$ & $V_{\text {meso }}\left(\mathrm{cm}^{3} \mathrm{~g}^{-1}\right)^{\mathrm{c}}$ & Pore size $(\mathrm{nm})^{\mathrm{d}}$ & STY $\left(\mathrm{kg} \mathrm{m}^{-3} \mathrm{~d}^{-1}\right)^{\mathrm{e}}$ \\
\hline C-ZIF-8 & $24 \mathrm{~h}$ & 1374 & 0.38 & 0.005 & 1.8 & 3.9 \\
\hline ZIF-8_D1 & $1 \mathrm{~min}$ & 1049 & 0.42 & 0.11 & 6.5 & $4.3 \times 10^{3}$ \\
\hline ZIF-8_C1 & $1 \mathrm{~min}$ & 664 & 0.44 & 0.23 & 13.8 & $1.29 \times 10^{4}$ \\
\hline ZIF-8_B1 & $1 \mathrm{~min}$ & 995 & 0.41 & 0.10 & 18.7 & $1.02 \times 10^{4}$ \\
\hline ZIF-8_A1 & $1 \mathrm{~min}$ & 566 & 0.66 & 0.11 & 32.8 & $5.91 \times 10^{3}$ \\
\hline ZIF-8_A5 & $5 \mathrm{~min}$ & 1551 & 0.83 & 0.31 & 19.1 & $1.23 \times 10^{3}$ \\
\hline ZIF-8_A10 & $10 \mathrm{~min}$ & 956 & 0.49 & 0.15 & 9.2 & l \\
\hline ZIF-8_A30 & $30 \mathrm{~min}$ & 930 & 0.48 & 0.26 & 7.4 & l \\
\hline
\end{tabular}

a) $S_{\mathrm{BET}}$ is the BET surface area calculated by applying the Brunauer-Emmett-Teller (BET) equation; b) $V_{\mathrm{t}}$ is the total pore volume; c) $V_{\text {meso }}$ is the mesopore volume; d) Pore size is the Barrett-Joyner-Halenda $(\mathrm{BJH})$ adsorption average pore width obtained from the adsorption branch of the $\mathrm{N}_{2}$ isotherm. e) STY is the space-time yield calculated based on the mass of active products.

a major challenge [6]. In this work, the sizes of mesopores in ZIF-8_At could be controlled by the synthesis time. As shown in Fig. 4b, the mesopore diameters of the as-synthesized ZIF- 8 materials systematically decrease from 40 to $15 \mathrm{~nm}$ depending on the synthesis time. Notably, the mesopores became more ordered, with pore sizes of $15 \mathrm{~nm}$, when the synthesis time was longer than $10 \mathrm{~min}$ (inset of Fig. 4b). This result can be attributed to initial aggregation of the nanoparticles into hierarchical porous phases with interparticle mesoporosity on the surfaces of the organic amine-template, followed by continuous aggregation of the nanoparticles into clumps. Subsequently, after removal of the amine template, smaller, well-ordered mesopores were formed [26], as confirmed by SEM and TEM (Figs 2 and 3). The porosities of the hierarchical porous ZIF-8_At samples are summarized in Table 1. On increasing the synthesis time from 1 to $30 \mathrm{~min}$, the BET surface areas $\left(S_{\mathrm{BET}}\right)$ of the ZIF-8_A $t$ samples decreased from $1566 \mathrm{~m}^{2} \mathrm{~g}^{-1}$ (for ZIF-8_A1) to $930 \mathrm{~m}^{2} \mathrm{~g}^{-1}$ (for ZIF-8_A30), but the total volume $\left(V_{\mathrm{t}}\right)$ increased from $0.66 \mathrm{~cm}^{3} \mathrm{~g}^{-1}$ (for ZIF-8_A1) to a maximum value of $0.83 \mathrm{~cm}^{3} \mathrm{~g}^{-1}$ (for ZIF-8_A5), and then decreased to $0.48 \mathrm{~cm}^{3} \mathrm{~g}^{-1}$ (for ZIF-8_A30). There may be a contribution from a larger crystal structure that leads to the decrease of $S_{\mathrm{BET}}$ and $V_{\mathrm{t}}[34,35]$. These analyses confirm that the porosities of hierarchical porous ZIF-8 materials can be tuned precisely by controlling the synthesis time.

\section{Other organic amines as supramolecular templates for hierarchical porous ZIF-8}

To confirm this concept of using organic amines to rapidly synthesize hierarchical porous ZIF-8 at RTP, three other organic amines (Table S1) were chosen as supra- molecular templates, as organic amines of different molecular sizes can be used to tune the porosity of hierarchical porous ZIF-8. The resulting products were named ZIF-8_X1, where $X$ represents the type of organic amine $(X=\mathrm{B}, \mathrm{C}$ or $\mathrm{D}$; $\mathrm{B}$ : N,N,N,N-tetramethylethylenediamine, C: $N, N$-dimethylbutylamine, and D: $N, N$-dimethylethanamine; Supplementary information). The XRD patterns of the resultant ZIF-8_X1 products agree with those of C-ZIF-8 and the simulated results (Fig. S3), confirming that these products are crystalline ZIF-8. The SEM and TEM images show obvious mesoporous structures in the ZIF- 8 _X1 products (Fig. S4). These results confirm that our synthesis method is quite solid, as hierarchical porous ZIF-8 materials were obtained within 1 min at RTP with each of the organic amines (Table 1). Notably, different crystal morphologies were observed for each of the ZIF-8_X1 ( $X=\mathrm{A}, \mathrm{B}, \mathrm{C}$, or D) samples depending on the type of organic amine (Fig. 2a and Fig. S4), which can be attributed to the different protonation abilities of the organic amines. The protonation ability may affect the nucleation rate of the ZIFs, resulting in the formation of various crystal morphologies [25,36]. As shown in Fig. S5, the $\mathrm{N}_{2}$ adsorption-desorption isotherms of ZIF-8_X1 exhibited an intermediate mode between type I and type IV with an apparent hysteresis loop, which indicates the coexistence of micropores and mesopores in these materials. However, differences were observed in adsorption capacity depending on the type of organic amine, implying that the porosity of hierarchical porous ZIF- 8 can be affected by the organic amine. The coexistence of micropores, mesopores, and macropores in the ZIF-8_X1 materials was further confirmed by the PSDs, as shown in Fig. S6a. In addition, we also noticed that the porosity of the ZIF materials depended on the 
type of organic amines, as shown in Fig. S6b. Furthermore, the average pore sizes of ZIF- 8 _ 1 become larger as the molecular size of the organic amine increased, as shown in Table 1. The observed relationship between pore size and molecular size may be related to the strong dependence of the mesopores on the volume of self-assembled organic amine, where an increased chain length leads to the formation of larger pores[7]. Hence, both the morphology and porosity of hierarchical porous ZIF-8 may be influenced by the type of organic amine.

\section{Chemical purity and thermal stability of hierarchical porous ZIF-8}

The Fourier transform infrared (FT-IR) spectra of ZIF$8 \_X 1(X=\mathrm{A}, \mathrm{B}, \mathrm{C}$, or $\mathrm{D})$ are in good agreement with that of C-ZIF-8, as shown in Fig. S7, which indicates that the ZIF-8_X1 samples are phase-pure [28]. However, in the ZIF-8_X1 ( $X=\mathrm{B}, \mathrm{C}$, or D) samples, a characteristic band is observed at around $3400 \mathrm{~cm}^{-1}$, which can be attributed to the presence of residual solvent (e.g., $\mathrm{H}_{2} \mathrm{O}$ or methanol) in these samples [37]. The content of $\mathrm{N}$ in ZIF-8_X1 $(X=\mathrm{B}, \mathrm{C}$, or $\mathrm{D})$ revealed by elemental analysis is much higher than that in C-ZIF-8 (Table S2), indicating that these samples contain a certain amount of residual organic amine-template. This result explains why the BET surface areas of these samples are lower than that of $\mathrm{C}$ ZIF-8 (Table 1). While organic amine residues could block a fraction of the pore channels, all the ZIF-8_Xt samples have much larger total pore volumes $\left(V_{\mathrm{t}}\right)$ and mesopore volumes $\left(V_{\text {meso }}\right)$ than C-ZIF-8 (Table 1$)$, which confirms the generation of mesoporosity in the ZIF-8_X1 samples. As shown in Fig. S8a, thermogravimetric analysis (TGA) of the hierarchical porous ZIF-8 materials and C-ZIF-8 reveals similar weight losses during the same thermal condition. In addition, the thermal stabilities of the ZIF- 8 materials were further investigated by analyzing these materials after exposure to air for 30 days (Fig. S8b). Similar changes were observed in these TGA curves, indicating that the hierarchical porous ZIF- 8 materials have good thermal stability.

\section{Extension of the supramolecular templating method to hierarchical porous ZIF-61 and ZIF-90}

Hierarchical porous ZIF-61 and ZIF-90 (named ZIF61_A and ZIF-90_A, respectively, where A indicates that diethanolamine was used as the supramolecular template) were also synthesized at RTP within 1 min using the organic amine-template method developed in this work (Supplementary information). The XRD pattern of assynthesized ZIF-61_A agrees with the simulated result
(Fig. S9), and the SEM and TEM images reveal a clear mesoporous structure in the ZIF-61_A powder, as shown in Fig. S10. In addition, all peak positions for ZIF-90_A match those in the simulated pattern (Fig. S11). As observed in Fig. S12, the SEM and TEM images of ZIF-90_A show a dense, uniform polyhedron structure of about $100 \mathrm{~nm}$ in diameter, with clear inter-particle voids. The TGA curves of ZIF-61_A and ZIF-90_A (Fig. S13) reveal that both samples have good thermal stability. These results indicate that our method allows various stable hierarchical porous ZIFs to be synthesized within $1 \mathrm{~min}$ at RTP.

\section{Mechanism for the rapid synthesis of hierarchical porous ZIFs}

Gross et al. [23] and Nordin et al. [24] found that triethylamine as a protonation agent can deprotonate ligands dissolved in organic solvents and initiate framework formation. To explore the mechanism by which rapid synthesis of hierarchical porous ZIFs occurs, we studied the molecular properties of triethylamine and the other four organic amines used in the present work using quantum chemistry (QC) calculations. All five organic amines contain an amine group and an alkyl chain (or hydroxyl moiety), as shown in Figs S14 and S15, respectively. The molecular geometries of the five organic amines were optimized at the B3LYP/6-311+G(d, p) level of theory using the Gaussian 09 program [38]. The molecular electrostatic potential (MEP) of each organic amine was calculated based on the optimized geometry using the DFT-B3LYP/6-31G* method. According to previous studies, the negative region (red) of the MEP map is related to electrophilic reactivity and the positive region (blue) to nucleophilic reactivity [39]. As shown in Fig. 5a, the negative region (red) of the MEP map of triethylamine is mainly located on the amine group, suggesting that this group is quite vulnerable to electrophilic attack. Furthermore, Fig. 5b shows that the distribution of the highest occupied molecular orbital (HOMO) of triethylamine, calculated at the B3LYP/6$31 \mathrm{G}^{*}$ level, is also mainly located on the amine group, where the energy of the HOMO is directly related to the ionization potential and characterizes the susceptibility of the molecule toward attack by electrophiles [40]. The MEPs and HOMO distributions of the four organic amines used in this work, which are presented in Figs S16 and S17, respectively, are also mainly located on the amine groups. These results led us to hypothesize that the protonation ability of the organic amines is mainly due to the presence of an amine group. A series of alkanes and 

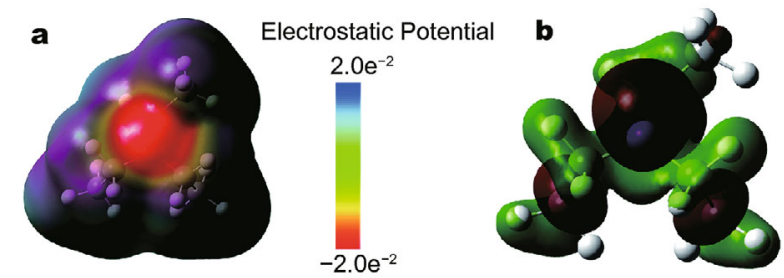

Figure 5 (a) Molecular electrostatic potential (MEP) map and (b) highest occupied molecular orbital (HOMO) surface of triethylamine $($ hydrogen atom $=$ white, carbon $=$ gray, and nitrogen $=$ blue $)$.

an alkyl alcohol (Table S3) were employed as supramolecular templates to verify this hypothesis, and no precipitation was observed within the same synthesis time. In addition, the MEP maps of the alkanes exhibit uniform distributions without apparent negative regions (red), as shown in Fig. S18. Although the MEP map and HOMO of the alkyl alcohol are mainly located on the hydroxyl group (Fig. S19), the experimental results indicated that the hydroxyl group cannot protonate the ligands. These findings confirm that the protonation role of the organic amines was mainly due to the amine group.

Based on the above results and literature precedent, a possible mechanism for the rapid synthesis of hierarchical porous ZIFs at RTP is illustrated in Scheme 1. In the initial stages, the organic amines act as protonation agents that deprotonate the organic ligands (e.g., 2-Im and methylimidazole (Im)), a key step in forming ZIFs rapidly [29]. Meanwhile, the protonated organic amines interact strongly with the ZIF ligands [7]. Then, the metal ion $\left(\mathrm{Zn}^{2+}\right)$ rapidly bonds to the deprotonated ligands (e.g., $2-\mathrm{Im}^{-}$and $\mathrm{Im}^{-}$) to achieve framework formation, with the organic amines acting as SDAs to direct the growth of the ZIF-template composites [34]. After removal of the organic amines, porous materials with hierarchical pores are obtained, in which the walls of the mesopores are composed of microporous frameworks [41].

\section{Enhancement of $\mathrm{CO}_{2} / \mathrm{CH}_{4}$ adsorption and selectivity}

Hierarchical porous MOF crystals incorporating micropores, mesopores, and macropores are desirable for fast diffusion and efficient uptake of guest molecules [42]. To investigate the adsorption properties of the activated ZIF8_A1 material, $\mathrm{CO}_{2}$ and $\mathrm{CH}_{4}$ adsorption isotherms were recorded at a broad range of high pressures at $298 \mathrm{~K}$. As shown in Fig. 6, the $\mathrm{CO}_{2}$ uptake of ZIF-8_A1 is 9.0 mmol g${ }^{-1}$, which is $73 \%$ higher than that of pristine C-ZIF-8 $\left(5.2 \mathrm{mmol} \mathrm{g}^{-1}\right)$ at $298 \mathrm{~K}$, and also easily surpasses the previously reported highest value of 5.5 mmol $\mathrm{g}^{-1}$ [43], under pressures up to $3 \mathrm{MPa}$. The en-

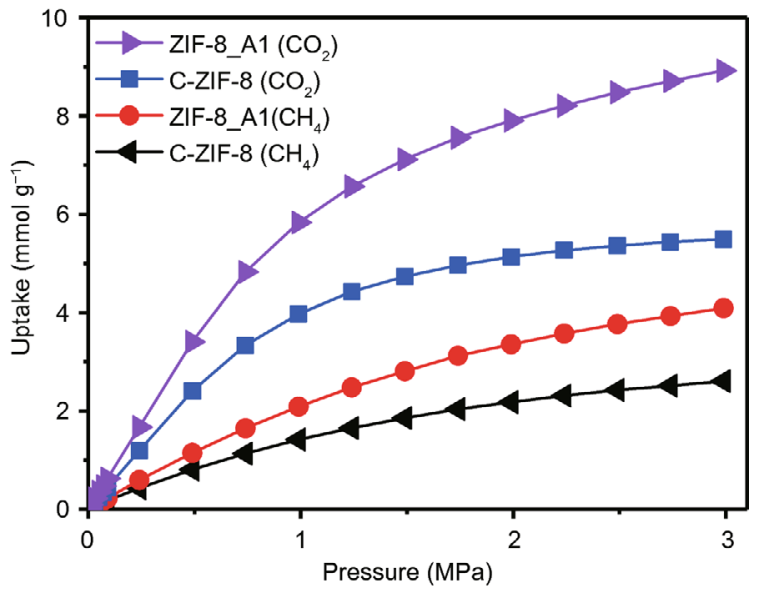

Figure $6 \mathrm{CO}_{2}$ and $\mathrm{CH}_{4}$ adsorption isotherms for C-ZIF-8 and ZIF-8_A1 samples at $298 \mathrm{~K}$.

hanced uptake capacity of $\mathrm{CO}_{2}$ could be ascribed to the larger pore volume of the ZIF-8_A1 sample [42]. More importantly, the loading of $\mathrm{CO}_{2}$ on ZIF-8_A1 is always higher than that on C-ZIF-8. As the micropore surface area of ZIF-8_A1 $\left(1158 \mathrm{~m}^{2} \mathrm{~g}^{-1}\right)$ is much smaller than that of C-ZIF-8 $\left(1355 \mathrm{~m}^{2} \mathrm{~g}^{-1}\right)$, the enhanced diffusion rate of ZIF-8_A1 can be attributed to the presence of many more mesopores and macropores which allow quick diffusion of the guest molecules to the active centers $[42,44]$. Similar profiles are observed for $\mathrm{CH}_{4}$ adsorption on the ZIF-8_A1 and C-ZIF-8 samples (Fig. 6). These results confirm that the introduction of mesopores and macropores not only improves the uptake capacity of gas molecules, but also increases the diffusion rate.

\section{Facile synthesis conditions and record STY for ZIF-8}

Energy consumption and production rates are important considerations in determining the cost and environmental impact (i.e., carbon footprint) of large-scale ZIF production $[9,45,46]$. Compared with conventional solvothermal methods with long crystalline times at HTP, our synthesis strategy allows the reaction to be completed within 1 min at RTP, which meets both the low-cost and environmental-friendly requirements for ZIF syntheses. In addition, facile synthesis conditions are highly popular in research, as they allow standard equipment to be used, including batch reactors and high-throughput setups suitable for ZIF discovery and optimization of crystallization [47]. The STYs of the hierarchical porous ZIF-8 and C-ZIF-8 samples synthesized in this work are summarized in Table 1. Although there have been a couple of reports on the STYs of conventional ZIFs [11], to the best of our knowledge, the STY of hierarchical porous ZIF-8 
has not yet been reported. For $1 \mathrm{~min}$ reactions, the STY varies between $4.3 \times 10^{3}$ and $1.29 \times 10^{4} \mathrm{~kg} \mathrm{~m}^{-3} \mathrm{~d}^{-1}$, depending on the type of organic amine. All these values easily surpass those of conventional solvothermal synthesis methods on the laboratory scale (C-ZIF-8, $3.9 \mathrm{~kg} \mathrm{~m}^{-3} \mathrm{~d}^{-1}$ ) and commercial ZIF-8 (Basolite Z1200, $100 \mathrm{~kg} \mathrm{~m}^{-3} \mathrm{~d}^{-1}$ ) [45], and are three times higher than the highest STY reported for C-ZIF-8 synthesized by the aerosol method $\left(3875 \mathrm{~kg} \mathrm{~m}^{-3} \mathrm{~d}^{-1}\right)$ [48]. The STY of hierarchical porous ZIF-8_C1 (up to $1.29 \times 10^{4} \mathrm{~kg} \mathrm{~m}^{-3} \mathrm{~d}^{-1}$ ) is a new record, which is comparable to those of Al-based MOFs that are currently produced on the ton-scale [49]. Although the STYs vary for the different organic amines, these values remain high, indicating that scale-up of the rapid room-temperature synthesis of hierarchical porous ZIFs to an industrial level is feasible. These results indicate that our synthesis strategy is of great promise for large-scale ZIF production with commercial viability and environmental sustainability.

\section{CONCLUSIONS}

In summary, a simple, green, and general method for rapidly synthesizing various hierarchical porous ZIFs (e. g., ZIF-8, ZIF-61, and ZIF-90) at RTP by employing an organic amine as a supramolecular template (organic amine-template) was demonstrated. The synthesis time can be as short as $1 \mathrm{~min}$, and the obtained hierarchical porous ZIF-8 materials possess three types of pores (micropores, mesopores, and macropores) together with good thermal stability. Moreover, the morphologies and porosities of the hierarchical porous ZIF-8 materials can be finely tuned by controlling the synthesis time or the type of organic amine. The organic amine played two roles during synthesis: (1) a protonation agent to deprotonate organic ligands, facilitating the formation of ZIF crystals, and (2) an SDA to direct mesopore/macropore formation. Furthermore, the protonation ability of organic amines is mainly due to the presence of the amine group. The hierarchical porous ZIFs exhibited significantly increased adsorption capacities for gas molecules owing to enhanced pore volumes and improved mass diffusion. Furthermore, a new record STY of $1.29 \times$ $10^{4} \mathrm{~kg} \mathrm{~m}^{-3} \mathrm{~d}^{-1}$ was achieved for the hierarchical porous ZIF-8 synthesis using the organic amine-template strategy developed in this work, which should be very promising for large-scale industrial synthesis of various hierarchical porous ZIFs for a wide variety of applications.

Received 19 July 2017; accepted 3 October 2017; published online 24 November 2017
1 Phan A, Doonan CJ, Uribe-Romo FJ, et al. Synthesis, structure, and carbon dioxide capture properties of zeolitic imidazolate frameworks. Acc Chem Res, 2010, 43: 58-67

2 Park KS, Ni Z, Côté AP, et al. Exceptional chemical and thermal stability of zeolitic imidazolate frameworks. Proc Natl Acad Sci USA, 2006, 103: 10186-10191

3 Li JR, Kuppler RJ, Zhou HC. Selective gas adsorption and separation in metal-organic frameworks. Chem Soc Rev, 2009, 38: 1477-1504

4 Lu G, Hupp JT. Metal-organic frameworks as sensors: a ZIF-8 based fabry-perot device as a selective sensor for chemical vapors and gases. J Am Chem Soc, 2010, 132: 7832-7833

$5 \mathrm{Xu} \mathrm{X,} \mathrm{Lu} \mathrm{Y,} \mathrm{Yang} \mathrm{Y,} \mathrm{et} \mathrm{al.} \mathrm{Tuning} \mathrm{the} \mathrm{growth} \mathrm{of} \mathrm{metal-organic}$ framework nanocrystals by using polyoxometalates as coordination modulators. Sci China Mater, 2015, 58: 370-377

6 Huang $\mathrm{H}, \mathrm{Li}$ JR, Wang $\mathrm{K}$, et al. An in situ self-assembly template strategy for the preparation of hierarchical-pore metal-organic frameworks. Nat Commun, 2015, 6: 8847

7 Bradshaw D, El-Hankari S, Lupica-Spagnolo L. Supramolecular templating of hierarchically porous metal-organic frameworks. Chem Soc Rev, 2014, 43: 5431-5443

$8 \mathrm{Na} \mathrm{K}$, Choi M, Ryoo R. Recent advances in the synthesis of hierarchically nanoporous zeolites. Microporous Mesoporous Mater, 2013, 166: 3-19

9 Julien PA, Mottillo C, Friščić T. Metal-organic frameworks meet scalable and sustainable synthesis. Green Chem, 2017, 19: 27292747

10 Tranchemontagne DJ, Hunt JR, Yaghi OM. Room temperature synthesis of metal-organic frameworks: MOF-5, MOF-74, MOF177, MOF-199, and IRMOF-0. Tetrahedron, 2008, 64: 8553-8557

11 Stock N, Biswas S. Synthesis of metal-organic frameworks (MOFs): routes to various MOF topologies, morphologies, and composites. Chem Rev, 2012, 112: 933-969

12 Wang B, Côté AP, Furukawa $\mathrm{H}$, et al. Colossal cages in zeolitic imidazolate frameworks as selective carbon dioxide reservoirs. Nature, 2008, 453: 207-211

13 Song L, Zhang J, Sun L, et al. Mesoporous metal-organic frameworks: design and applications. Energ Environ Sci, 2012, 5: 75087520

14 Kim MK, Kim D, Seo JY, et al. Nanostructured ZnO as a structural template for the growth of ZIF-8 with tunable hierarchical porosity for $\mathrm{CO}_{2}$ conversion. CrystEngComm, 2017, 19: 4147-4151

$15 \mathrm{Wu}$ YN, Zhou M, Zhang B, et al. Amino acid assisted templating synthesis of hierarchical zeolitic imidazolate framework-8 for efficient arsenate removal. Nanoscale, 2014, 6: 1105-1112

16 Kim D, Kim DW, Hong WG, et al. Graphene/ZIF-8 composites with tunable hierarchical porosity and electrical conductivity. J Mater Chem A, 2016, 4: 7710-7717

17 Peng L, Zhang Z, Xue WG, et al. Highly mesoporous metal-organic framework assembled in a switchable solvent. Nature Commun, 2014, 5: 4465

18 Zeng M, Chai Z, Deng X, et al. Core-shell CdS@ZIF-8 structures for improved selectivity in photocatalytic $\mathrm{H}_{2}$ generation from formic acid. Nano Res, 2016, 9: 2729-2734

19 Bux H, Liang F, Li Y, et al. Zeolitic imidazolate framework membrane with molecular sieving properties by microwave-assisted solvothermal synthesis. J Am Chem Soc, 2009, 131: 1600016001

20 Seoane B, Zamaro JM, Tellez C, et al. Sonocrystallization of zeolitic 
imidazolate frameworks (ZIF-7, ZIF-8, ZIF-11 and ZIF-20). CrystEngComm, 2012, 14: 3103-3107

21 Beldon PJ, Fábián L, Stein RS, et al. Rapid room-temperature synthesis of zeolitic imidazolate frameworks by using mechanochemistry. Angew Chem Int Ed, 2010, 49: 9640-9643

22 Zhuang JL, Ceglarek D, Pethuraj S, et al. Rapid room-temperature synthesis of metal-organic framework HKUST-1 crystals in bulk and as oriented and patterned thin films. Adv Funct Mater, 2011, 21: $1442-1447$

23 Gross AF, Sherman E, Vajo JJ. Aqueous room temperature synthesis of cobalt and zinc sodalite zeolitic imidizolate frameworks. Dalton Trans, 2012, 41: 5458-5460

24 Nordin NAHM, Ismail AF, Mustafa A, et al. Aqueous room temperature synthesis of zeolitic imidazole framework 8 (ZIF-8) with various concentrations of triethylamine. RSC Adv, 2014, 4: 33292-33300

25 Cravillon J, Nayuk R, Springer S, et al. Controlling zeolitic imidazolate framework nano- and microcrystal formation: insight into crystal growth by time-resolved in situ static light scattering. Chem Mater, 2011, 23: 2130-2141

26 Hirai K, Furukawa S, Kondo M, et al. Sequential functionalization of porous coordination polymer crystals. Angew Chem Int Ed, 2011, 50: 8057-8061

27 Kim MK, Kim D, Seo JY, et al. Nanostructured ZnO as a structural template for the growth of ZIF-8 with tunable hierarchical porosity for $\mathrm{CO}_{2}$ conversion. CrystEngComm, 2017, 19: 4147-4151

28 Cao S, Gody G, Zhao W, et al. Hierarchical bicontinuous porosity in metal-organic frameworks templated from functional block cooligomer micelles. Chem Sci, 2013, 4: 3573

29 Cravillon J, Schröder CA, Nayuk R, et al. Fast nucleation and growth of ZIF-8 nanocrystals monitored by time-resolved in situ small-angle and wide-angle X-ray scattering. Angew Chem, 2011, 123: 8217-8221

30 Wang Z, Zhang Q, Kuehner D, et al. Green synthesis of 1-2 nm gold nanoparticles stabilized by amine-terminated ionic liquid and their electrocatalytic activity in oxygen reduction. Green Chem, 2008, 10: 907-909

31 Zhu Y, Hua Z, Zhou J, et al. Hierarchical mesoporous zeolites: direct self-assembly synthesis in a conventional surfactant solution by kinetic control over the zeolite seed formation. Chem Eur J, 2011, 17: 14618-14627

32 Junggeburth SC, Schwinghammer K, Virdi KS, et al. Towards mesostructured zinc imidazolate frameworks. Chem Eur J, 2012, 18: $2143-2152$

33 Sing KS. Reporting physisorption data for gas/solid systems with special reference to the determination of surface area and porosity (recommendations 1984). Pure Appl Chem, 1985, 57: 603-619

34 Sun LB, Li JR, Park J, et al. Cooperative template-directed assembly of mesoporous metal-organic frameworks. J Am Chem Soc, 2011, 134: $126-129$

35 Walton KS, Snurr RQ. Applicability of the BET method for determining surface areas of microporous metal-organic frameworks. J Am Chem Soc, 2007, 129: 8552-8556

36 Rived F, Canals I, Bosch E, et al. Acidity in methanol-water. Anal Chim Acta, 2001, 439: 315-333

37 Naumann D, Helm D, Labischinski H, et al. The characterization of microorganisms by Fourier-transform infrared spectroscopy (FT-IR). Modern techniques for rapid microbiological analysis, 1991, 43-96

38 Liu B, Tan Y, Ren Y, et al. Fabrication of a hierarchically structured beta zeolite by a dual-porogenic surfactant. J Mater Chem, 2012, 22: 18631

39 Liu B, Zheng L, Zhu Z, et al. Hierarchically structured Beta zeolites with intercrystal mesopores and the improved catalytic properties. Appl Catal A-General, 2014, 470: 412-419

40 Khaled KF, Fadl-Allah SA, Hammouti B. Some benzotriazole derivatives as corrosion inhibitors for copper in acidic medium: experimental and quantum chemical molecular dynamics approach. Mater Chem Phys, 2009, 117: 148-155

41 Qiu LG, Xu T, Li ZQ, et al. Hierarchically micro- and mesoporous metal-organic frameworks with tunable porosity. Angew Chem Int Ed, 2008, 47: 9487-9491

42 Mao Y, Chen D, Hu P, et al. Hierarchical mesoporous metalorganic frameworks for enhanced $\mathrm{CO}_{2}$ capture. Chem Eur J, 2015, 21: 15127-15132

43 Liu D, Wu Y, Xia Q, et al. Experimental and molecular simulation studies of $\mathrm{CO}_{2}$ adsorption on zeolitic imidazolate frameworks: ZIF8 and amine-modified ZIF-8. Adsorption, 2013, 19: 25-37

44 Wee LH, Lescouet T, Ethiraj J, et al. Hierarchical zeolitic imidazolate framework- 8 catalyst for monoglyceride synthesis. ChemCatChem, 2013, 5: 3562-3566

45 Czaja AU, Trukhan N, Müller U. Industrial applications of metalorganic frameworks. Chem Soc Rev, 2009, 38: 1284-1293

46 Vellingiri K, Kumar P, Kim KH. Coordination polymers: challenges and future scenarios for capture and degradation of volatile organic compounds. Nano Res, 2016, 9: 3181-3208

47 Stock N. High-throughput investigations employing solvothermal syntheses. Microporous Mesoporous Mater, 2010, 129: 287-295

48 Munn AS, Dunne PW, Tang SVY, et al. Large-scale continuous hydrothermal production and activation of ZIF-8. Chem Commun, 2015, 51: 12811-12814

49 Gaab M, Trukhan N, Maurer S, et al. The progression of Al-based metal-organic frameworks - from academic research to industrial production and applications. Microporous Mesoporous Mater, 2012, 157: 131-136

Acknowledgements This work was supported by the National Natural Science Foundation of China (21576094), SRFDP (20130172110012) and the Fundamental Research Funds for the Central Universities (2015ZM046)

Author contributions Original idea was conceived by Duan CX, Li C and Xi HX. Experiment design and data analysis were performed by Duan CX, Li FE and Liu ZW. Calculation and computational simulations were performed by Duan CX. Manuscript was drafted by Duan CX, Xiao J, and Xi HX. All authors discussed the results and commented on the manuscript.

Conflict of interest The authors declare that they have no conflict of interest.

Supplementary Information Experimental section and other data are available in the online version of this paper. 


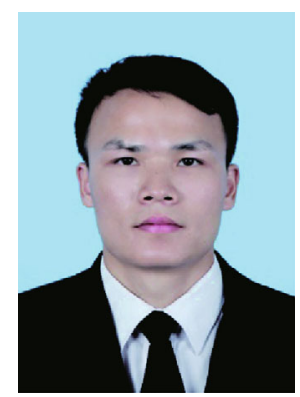

Chongxiong Duan is a Ph.D candidate at South China University of Technology (SCUT), under the supervision of Prof. Hongxia Xi. His research focused on the synthesis of hierarchical porous nanomaterials.

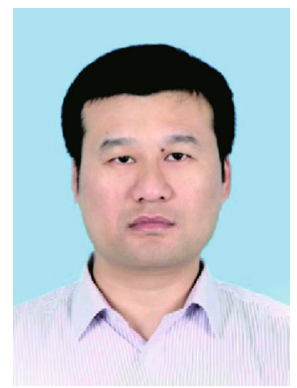

Chao Li received his BSc, MSc and PhD degrees in chemical engineering from Northwest University in 2007, Central South University in 2010, and SCUT in 2016, respectively. During 2012-2014, he worked as a visiting scholar at the Department of Chemical Engineering, the University of Massachusetts-Amherst, USA. In 2016, he joined SCUT as a postdoc member. His research interests include synthesis and characterization of functional nanoporous materials (zeolite, MOF, silica, carbon), heterogeneous catalysis and green chemistry.

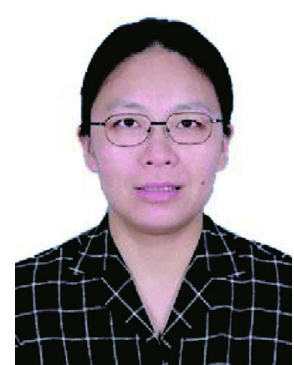

Hongxia Xi received her PhD degree in Chemical Engineering from SCUT in 1996. She then worked as a post-doctor for two years at Sun Yat-sen Unviersity, as a visiting scholar for one year at Savoie University, France, and as a senior visiting scholar for six months at The State University of New Jersey, USA. She is currently a professor of chemical engineering at SCUT. Her research interests focus on the development of porous materials and their application.

\section{快速常温合成高空时产率的多极孔ZIFs材料}

段崇雄, 李非儿, 肖静, 柳泽伟, 李超", 奚红霞

摘要 在常温下快速合成具有多级孔结构的ZIFs材料具有重要的意义: 一方面, 多级孔道结构能有效地增加分子扩散速率、降低其传质阻 力, 这对于涉及大分子参与的反应具有重要意义, 扩大了材料的应用范围; 另一方面, 在常温下快速合成ZIFs材料不仅能大幅降低能源消 耗, 并且能极大地提高生产效率, 是一种绿色高效的工艺路线. 本文采用有机胺类作为模板, 首次在常温下快速合成具有高空时产率的 3 种 多级ZIFs材料(ZIF-8, ZIF-61 以及ZIF-90), 材料的合成时缩短至1分钟, 同时兼具微孔, 介孔, 大孔结构. 此外, ZIFs产物的形貌和多孔性质可 以通过调节胺模板的类型或者合成时间来调控. 更为重要的是, ZIFs材料的空时产率高达 $1.29 \times 10^{4} \mathrm{~kg} \mathrm{~m}^{-3} \mathrm{~d}^{-1}$, 比之前报导过的值高出3倍, 这为ZIFs材料的商业化生产提供了可能. 最后, 我们通过对有机胺类分子进行计算机模拟, 再结合之前文献报导的结果, 得到了一般性的 合成机理: 一方面有机胺作为质子化剂使得有机配体去质子化, 被质子化的配体迅速地和金属离子结合形成金属有机骨架; 另一方面, 有 机胺作为模板被用来引导介孔和大孔结构的形成. 